\title{
Design of a new-concept conical positive displacement slurry pump for continuous de-clogging
}

\author{
G. Vasileiou ${ }^{1}, N$. Rogkas ${ }^{1}, S$. Tsolakis ${ }^{1}, V$. Spitas ${ }^{1}$, and $P$. Zalimidis $^{2}$ \\ ${ }^{1}$ School of Mechanical Engineering, National Technical University of Athens - NTUA \\ ${ }^{2}$ Department of Mechanical Engineering Educators, School of Pedagogical and Technological Education - ASPETE
}

\begin{abstract}
Slurry pumps are extensively used in the construction industry while positive displacement screw pumps are used in most mobile concrete pump applications. The aggregate size is known to significantly affect pump performance in terms of clogging. Large aggregates tend to be trapped against the stator-rotor interface, blocking the continuous and smooth operation of the screw pump. In order to avoid the development of excessive stress values able to damage the rotor-stator mechanism of the pump, the typical de-clogging mechanism deployed by most positive displacement screw slurry pumps includes reversing the rotation of the pump driving motor thus allowing the aggregates to be carried away with the mixture, so that the pump can soon resume its operation. This procedure causes frequent start-stops of the pump resulting in dis-continuation of the pumped mixture lasting a few seconds, that while being of little importance in most construction applications, can be of significance in applications requiring higher levels of accuracy and continuous mixture flow. In the context of this work, a novel concept of positive displacement screw slurry pump is presented, including a continuous de-clogging mechanism, without the need to reverse the rotation of the driving motor. This de-clogging operation is achieved through the modification of the geometry of both the rotor and stator introducing a conical form along the axial direction. This configuration of the rotor-stator, allows for small displacements along the axial direction, which in turn increases the size of the cavities facilitating the de-clogging of the pump. Variable pitch is also introduced to both the rotor and stator in order to ensure constant mass flow of the mixture throughout the length of the screw pump covering for the velocity increase as a result of the conical geometry. The axial movement of the rotor in relation to the fixed stator, is achieved through the elastic support of the rotor in the axial direction, that allows for small axial displacements, when stresses induced from trapped aggregates exceed the stiffness of the support. The proposed concept comprises a passive real-time declogging mechanism that greatly reduces pump idle time compared to the conventional mechanism described earlier, providing smoother operation and stable mass flow of the mixture.
\end{abstract}

\section{Introduction}

Progressive cavity pumps (PCPs) are extensively used in a variety of applications including water and waste handling, deliquification of gas wells [1], oil production (in shallow oil wells) as well as the construction industry, given their ability to efficiently handle viscous liquids and solid materials (usually granular solids or mixtures of liquids and solids) while having relatively low surface profile compared to other positive displacement pumps used in similar applications. Due to their extensive use in gas and oil applications, progressive cavity pumps are thoroughly investigated in such applications, describing their function using analytical methods as performed by Nguyen et al. [2] and Zhou et al. [3] who performed three-dimensional analysis on progressive cavity pumps used in the gas and oil industry using semi-analytical models and Finite Element Analysis (FEA). Chen et al. [4] investigated the correlation of the volumetric efficiency of PCPs with the pressure difference using FEA and fluid-structure interaction methods followed by experimental data, identifying two leakage mechanisms. Other works include presentation of various types of PCPs used in the oil industry [5] as well as examination of common failure modes appearing in such applications such as adhesive failure between the elastomer of the stator and the steel housing [6] and design optimization of PCPs for use in abrasive environments [7].

In the context of this paper however, PCPs used in the construction industry are examined in terms of their main operational issue being clogging during operation. Cement slurries are mixtures consisted of cement, water, various additives (related to the concrete properties) and fine or coarse aggregates (i.e. gravel) depending on the application. During operation, aggregates are trapped at the stator - rotor interface resulting in the development of high stress values able to damage the stator - rotor mechanism of the pump. These extreme stress values also result in high torque requirements of the driving unit

Corresponding author: gvasileiou@mail.ntua.gr 
that in turn needs to cease operation in order to ensure its structural integrity. The lack of scientific works related to this topic is justified by the fact that construction industry PCPs are considered to have finite lives turning the attention of the manufacturers towards fast and easy disassembly and spare part sales [8]. A common practice used worldwide as a de-clogging mechanism includes driving motor rotation reversal - as soon as high torque requirements are developed - so that the trapped aggregates can be carried away with the mixture allowing the pump to resume its operation. This procedure (rotation reversal and restoration) is a frequent phenomenon in most construction applications and can last for up to a few seconds, leading to intermittent pump operation including slurry mass flow discontinuation and unstable operation. These drawbacks are negligible in most common construction industry applications, nevertheless are deemed significant in higher accuracy applications that require stable slurry flow rate and accuracy, where the frequent start - stops of the pump mechanism can also induce dynamic oscillations that add to the aforementioned problems. Such applications include concrete $3 \mathrm{D}$ printing in the construction industry (large scale applications) and 3D printing of high accuracy ceramic components (small to medium scale applications).

The present work introduces a novel design of the pump de-clogging mechanism that does not include rotation reversal opting for the modification of the stator - rotor geometry instead, by the introduction of a conical shape of the two components along the axial direction and the redesign of the coupling mechanism between the pump and the driving unit. The conical geometry ensures that the rotor of the PCP is allowed to move in the axial direction in the range of a few millimetres in order to increase the cavities of the stator - rotor interface thus allowing for additional space for the trapped aggregates to get carried away. The redesign of the coupling mechanism serves two distinct functions, namely providing the rotor with the required pretension that prevents axial movement during normal operation (i.e. as a result of the pressure difference between the inlet and the outlet of the PCP) and secondly allowing the axial movement and in consequence the de-clogging operation when extreme axial loads are developed as a result of trapped aggregates.

\section{Progressive cavity pump design}

Progressive cavity pumps (PCPs) represent a modified, more complex design of the Moineau pumps [9] in order to counter backflow. The main components of a PCP are the stator and rotor shown assembled in Figures $(1,2)$.

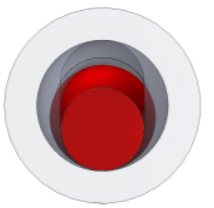

Fig. 1. Front view of a PCP with lobe ratio 2:1. The rotor is shown in red colour and the stator is shown in transparent grey.

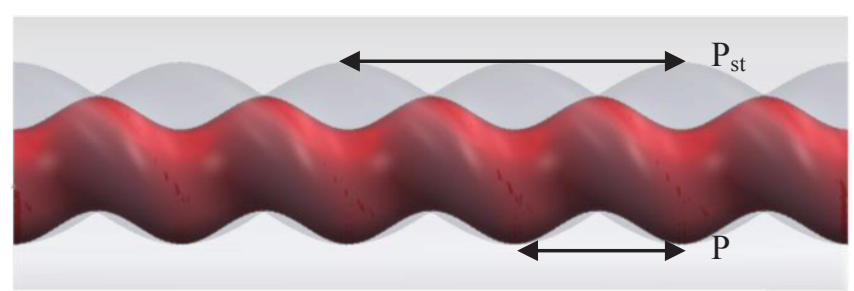

Fig. 2. Demonstration of a PCP side view. The rotor is shown in red colour and the stator is shown in transparent grey.

A major characteristic of the PCPs is the lobe ratio appearing in Fig. $(1,2)$ that relates the number of the lobes of the stator to the lobes of the rotor. The current study deals with PCPs with lobe ratio 2:1 (single lobe PCPs) since single lobe PCPs represent the vast majority of low and medium range construction applications. Saveth and Klein [10] described the basic design and performance characteristics of PCPs in relation to their geometry. Works on pressure distribution [11] and their effect on their performance and lifespan, modelling, simulation and optimization of PCPs in terms of their flow distribution and performance metrics [12 - 14], relate the geometrical characteristics of PCPs to the respective properties.

\subsection{Progressive cavity pump kinematics}

Motion analysis of PCPs exhibits a complex motion of the rotor relative to the stator. PCPs are generally categorised in two major classes in terms of their kinematics. Being geometrically similar to screw pumps, the male helix/ thread of the rotor is rotating inside the female helix/ thread of the stator. In the case of the simple Moineau pump, the stator consists of a hollow cylinder inside which the rotor is free to rotate. However, given the thread form of the stator, in order for the rotor to achieve its rotation, a second degree of freedom must be allowed to move.

The most common type of construction applications PCPs allows for the translation of the rotor normal to the axis of rotation. Therefore, the rotor shown in Fig. (2) oscillates vertically while rotating in order to avoid interference with the stator. This oscillatory movement is passively created through the interaction of the two solids (stator - rotor). The use of a universal joint is mandatory in order to transmit power from the power unit that is fixed in place as shown schematically in Figure 3. The second category of PCPs include the linear translation of the rotor collinearly to its axis of rotation. This oscillatory motion is again passively controlled and derived from the loads developed in the stator - rotor interface. In order for the rotor to move axially, split couplers are used allowing for the axial translation through spline connections between the drive unit and the rotor. This category is rare in commercial PCPs and is displayed schematically in Figure (4).

As far as the de-clogging mechanism is concerned, none of the layouts described provides a passive way to de-clog the pump when aggregates are trapped, since the secondary motions (vertical or axial oscillation 
correspondingly) derive from the stator - rotor interface in real time and require the rotation of the rotor in order to materialise. However, at any moment when an aggregate is trapped, the input torque cannot lead to the rotation of the rotor and therefore none of the vertical or linear oscillations can be performed.

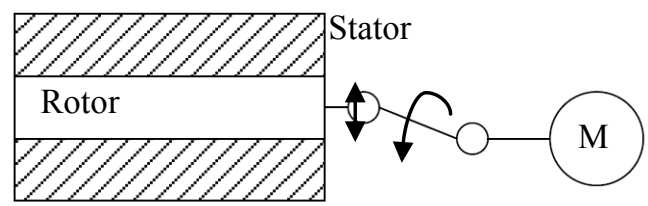

Fig. 3. Use of universal joint to achieve vertical oscillation.

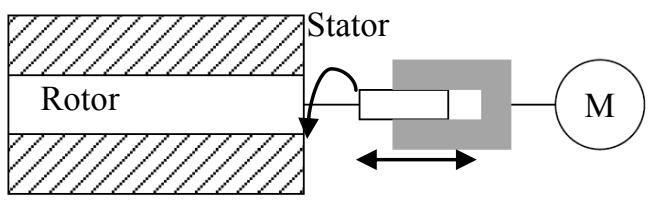

Fig. 4. Use of linear joint to allow axial oscillation.

\subsection{Basic geometric and operational equations}

The basic characteristics of the single lobe PCPs include the rotor diameter, pitch and eccentricity. All three variables affect the operational properties of the PCP such as volumetric flow rate, operational pressure, mixture velocity and power requirements.

Referring to the pitch $(\mathrm{P})$ of the PCP that corresponds to the pitch of the rotor as displayed in Fig. (2). For the single lobe PCPs examined in this paper, the stator pitch $\left(\mathrm{P}_{\mathrm{st}}\right)$ is double that of the rotor. Figures $(5,6)$ show the basic dimensions of the rotor - stator mechanism

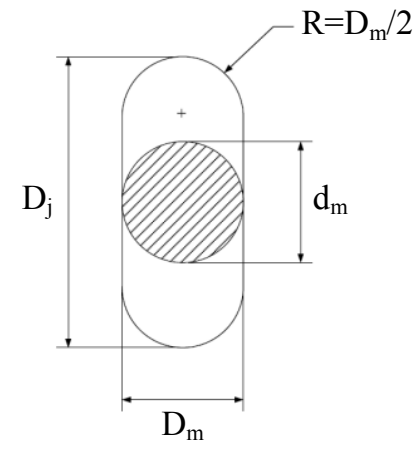

Figure 5: Dimensions of the rotor-stator mechanism (single lobe).

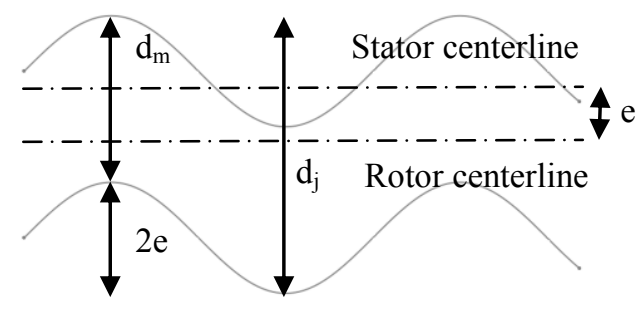

Fig. 6. PCP rotor basic dimensions.

The eccentricity (e) is an important characteristic of the stator - rotor mechanism and refers to the distance between the axis of the two components as shown in Fig. (6). The set of equations describing the basic geometrical features of the stator - rotor mechanism is given in Equations (1-4) below.

$$
\begin{gathered}
d_{m}=2 r \\
d_{j}=d_{m}+2 e \\
D_{m}=d_{m} \\
D_{j}=D_{m}+4 e
\end{gathered}
$$

Equations (1-4) describe the nominal dimensions of the stator - rotor mechanism. In reality however, PCPs are characterised by the fit of the stator - rotor mechanism, declared as the fit tolerance (c) which can be either positive (loose fit) or negative (tight fit). The fit tolerance can be of significance in terms of leakage or backflow existence as well as the torque requirements of the PCP. In order to incorporate fit values in the design and analysis of PCPs, we consider the total fit value as part of the stator dimensions whereas the rotor is considered to have nominal dimensions. Therefore, the actual stator dimensions are given as follows:

$$
\begin{aligned}
D_{m} & =D_{m 0}+2 c \\
D_{j} & =D_{j 0}+2 c
\end{aligned}
$$

The specific flow rate (q) of the pump calculates the volumetric flow rate per revolution, while the theoretical volumetric flow rate $\left(\mathrm{Q}_{0}\right)$ is calculated through Equation (7) by multiplying the cavity volume $\left(\mathrm{V}_{\text {cav }}\right)$ with the rotational speed (n). The cavity volume is calculated through multiplication of the cavity section area that is constant throughout the length of the PCP and is equal to $\mathrm{A}=4 \mathrm{ed}_{\mathrm{m}}$ as can be calculated using Fig. (5) and Eq. (4) with the pitch of the stator $\left(\mathrm{P}_{\mathrm{st}}=2 \mathrm{P}\right)$.

$$
Q_{0}=V_{c a v} n
$$

The actual flow rate of the pump differs from the theoretical value $\left(\mathrm{Q}_{0}\right)$ due to leakages. Calculation of the leakage/ backflow of the pump is used to calculate the volumetric efficiency of the pump.

$$
\eta=Q / Q_{0}
$$

where $\mathrm{Q}$ is the actual volumetric flow rate in SI units. As in most positive displacement pumps, the torque needed is derived by the volumetric flow and the pressure difference between the pump ends as in Equation (9).

$$
T=Q \Delta p
$$

\section{De-clogging mechanism design}

As stated earlier, the scope of this work includes the design of a novel PCP for continuous passive declogging with the incorporation of two key components, namely the geometrical modification of the usual single lobe PCP design through the introduction of conical geometry and the design of a novel adjustable coupler that allows the rotor to move axially thus freeing space for trapped aggregates to be carried through along with the mixture. 


\subsection{Conical geometry}

In order to design the novel PCP geometry a commercial PCP (D6 Power Putzmeister) was used as a reference point, in order for the designed PCP to have similar volumetric flow rate. The geometry of both the rotor and stator were measured in a CMM machine (see Figure 7).

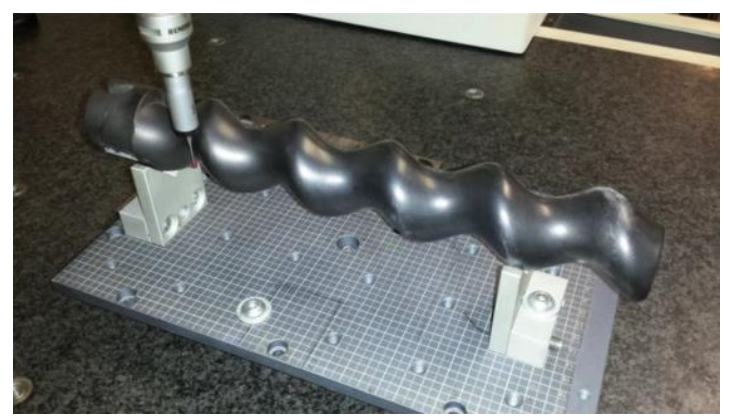

Figure 7. CMM measurement of commercial rotor dimensions

The measured dimensions are given in Table 1 below.

Table 1. Measured commercial PCP dimensions.

\begin{tabular}{|c|c|}
\hline Dimension & $\mathbf{m m}$ \\
\hline $\mathrm{d}_{\mathrm{m}}$ & 38 \\
\hline $\mathrm{P}$ & 55 \\
\hline $\mathrm{e}$ & 5.5 \\
\hline $\mathrm{L}$ & $275(=5 \mathrm{P})$ \\
\hline
\end{tabular}

The theoretical specific volumetric flow rate of the pump is calculated at $\mathrm{q}=92 \times 10^{-3} \mathrm{lt} / \mathrm{rev}$. The specific volumetric flow rate is a function of all three parameters measured in Table 1. In order to simplify the parametrization of the problem, the minimum rotor diameter $d_{m}$ is set to be constant at $38 \mathrm{~mm}$. The product (eP) needs to remain constant throughout the length of the PCP in order to maintain similar specific volumetric flow rate as in the measured PCP. Therefore, the product is calculated as $\mathrm{eP}=302.5$. Using $\mathrm{eP}=$ constant, the mean axial velocity of the mixture changes with the pitch $(\mathrm{P})$ as seen in Equation (10).

$$
u_{m}=Q_{0} / A=V_{\text {cav }} n / A=A P n / A=P(x) n
$$

The outlet of the designed PCP is set to have the same pitch (and consequently the same eccentricity) as the measured PCP. Five full revolutions of the helix are also used. The cone half-angle is set at one degree expanding towards the input of the PCP. The designed PCP is shown in Figure 8.

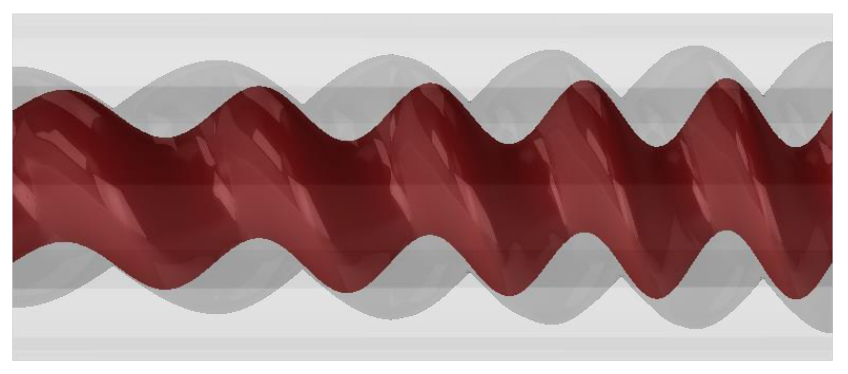

Fig. 8. Designed conical, variable pitch PCP.

The derived form of the novel PCP shown in Figure (8) maintains the same theoretical

Introducing increased radial clearance to $0.5 \mathrm{~mm}$ (larger than the average $0.2 \mathrm{~mm}$ mentioned earlier in this paper) we can achieve axial displacement in the order of $1.5 \mathrm{~mm}$. In consequence, the innovative PCP presented can passively carry mixtures containing medium size aggregates approximately up to $1.2 \mathrm{~mm}$ in diameter.

\subsection{Modified axial coupler}

In order to complete the proposed design of the passive de-clogging mechanism of the described PCP the design of the axial coupler has to be described also. As already mentioned, the coupler needs to allow the rotor of the PCP to perform small axial displacements in order for trapped aggregates to be carried away by the flowing mixture. The proposed coupler is the embodiment design of a Kelvin - Voight single degree of freedom (DOF) model consisting of a tuneable spring connected in parallel to a damper. The use of a damper is mandatory in order to avoid oscillatory motion of the rotor after the withdrawal phase in order to minimize the time needed to resume normal operation conditions. The tuneable spring serves a double purpose. It can be adjusted according to the type and hardness of the aggregates inside the mixture as well as according to the weight content of large aggregates (near the de-clogging capacity of the PCP) of the mixture. The spring of the coupler pre-stresses the rotor to its nominal position in order to assure proper functionality and minimum leakage as a result of the surface pressure on the rotor stator interface. The simple harmonic (single DOF) oscillator that describes the designed coupler provides the ability to adjust the PCP performance (response in terms of withdrawal and return time, minimum load required for axial displacement etc) according to the mixture characteristics (i.e. mixture density, mean aggregate size). Therefore, the coupler can be tailor made application-wise to perfectly fit the requirements. Moreover, the coupler contains mechanical hard stops to ensure the rotor cannot exceed the required axial displacements. Finally, it can easily be incorporated into both PCP kinematic layouts described in paragraph 2.1. Figures 9 schematically describes the layout of the designed coupler in normal operation and during withdrawal. The outer components of the coupler are connected through a spline in order to be able to transmit the torque provided by the drive unit. 


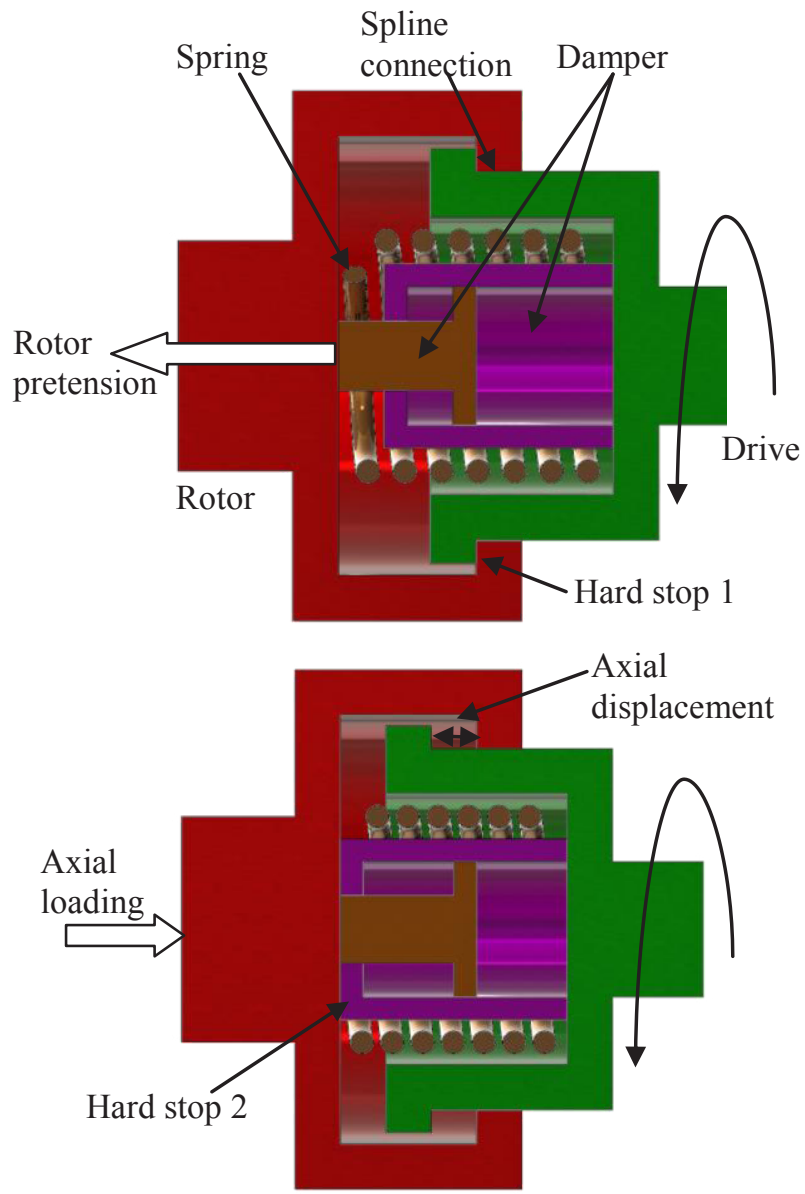

Fig. 9. Schematic layout of the designed coupler. Upper: during normal operation, the spring provides axial pretension to the rotor of the PCP. Lower: the coupler moves axially along with the rotor as a result of the loads induced by trapped aggregates.

\subsection{De-clogging mechanism overview}

Figures 10-12 depict a complete operation cycle of the novel PCP design that incorporates the passive declogging mechanism. Figure (10) shows the normal operation of the PCP at the moment when an aggregate is trapped between the rotor - stator interface.

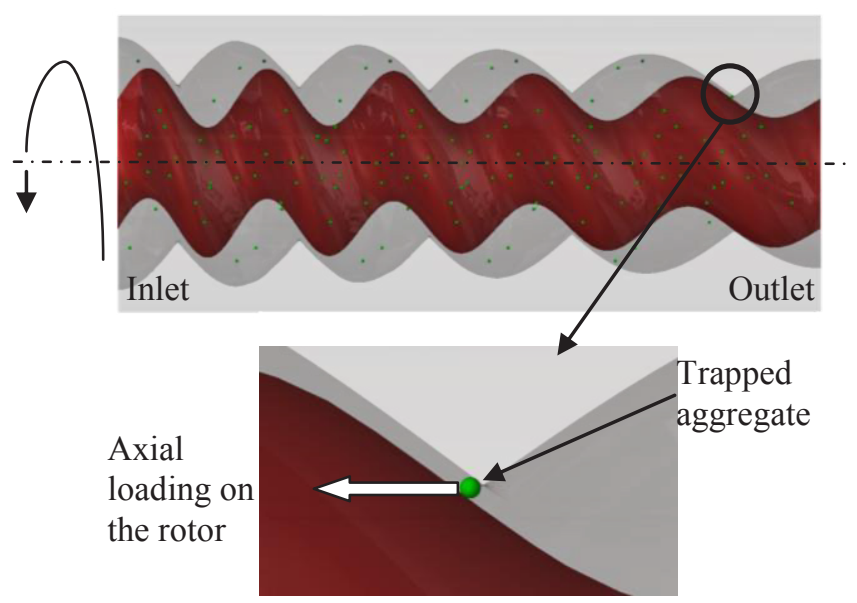

Fig. 10. The PCP operates normally at the moment when an aggregate is trapped between the stator - rotor interface resulting in the development of axial load on the rotor (detail view).
Entrapment of the aggregate shown in Fig. (10) would result the PCP being clogged thus initiating the de-clogging mechanism that includes rotation reversal of the drive unit. However, the novel PCP that is presented in this work will act as explained earlier. Specifically, the rotor will withdraw along with the coupler freeing space for the aggregate to be carried away with the mixture as shown in Figure 11.

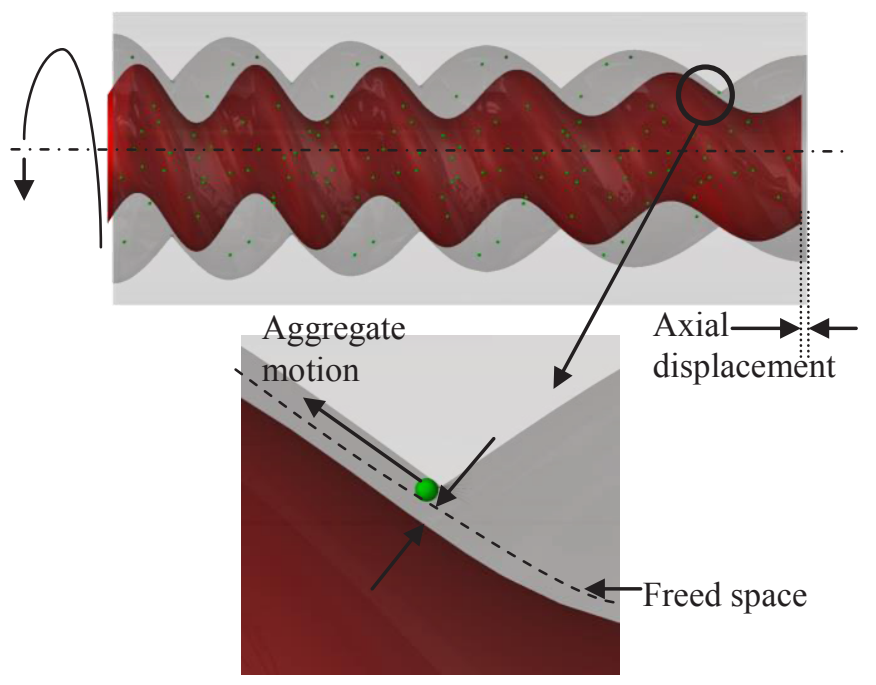

Fig. 11. Rotor withdrawal (displacement to the left). Free space is created for the trapped aggregate to be carried away with the rest of the mixture (detail view).

As soon as the rotor withdraws and free space is created, the trapped aggregate is carried away with mixture, allowing the rotor to advance to its nominal position aided by the spring tension and resume its operation. The restoration of the rotor position is shown in Figure 12. It has to be mentioned that throughout the de-clogging procedure, the drive unit continues to rotate and therefore the flow rate remains almost constant. Increasing the clearance of the stator - rotor interface leads to temporal increase of the leakage of the PCP, however due to the time period of the de-clogging action being very small the flow rate fluctuation caused is deemed negligible compared to the rotation reversal performed in commercial PCPs.

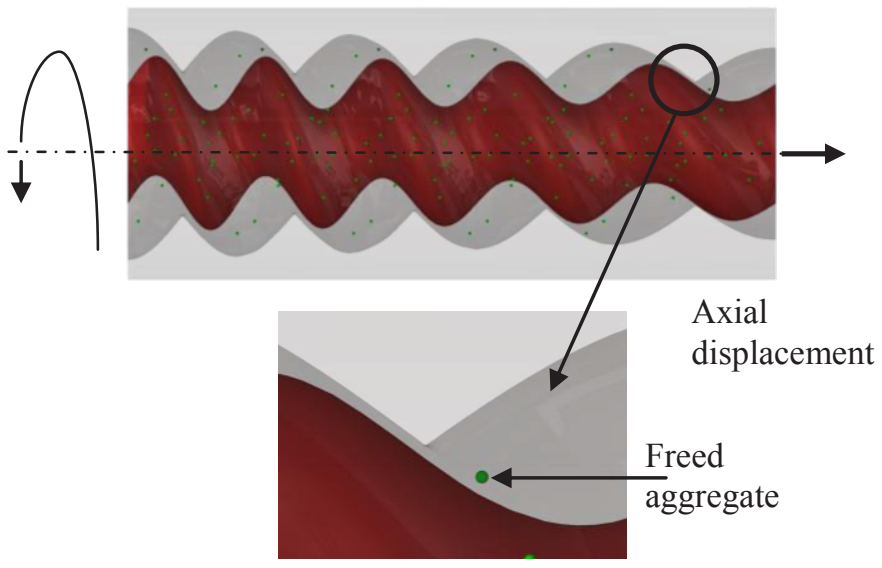

Fig. 12. The rotor advances to its nominal position (axial displacement to the right), while the aggregate is carried away with mixture. 


\section{Discussion}

The presented work described the design of an innovative progressive cavity pump that incorporates a passive de-clogging mechanism consisted of the modified conical, variable pitch stator - rotor geometry and the coupler that facilitates axial displacement of the rotor relative to the stator. The geometrical properties of the PCP were presented along with the description of the coupler layout. The steps of the de-clogging procedure were also described in detail.

The findings of this work were promising and point towards possible future applications of the proposed design. Nevertheless, future work on the scientific area of PCPs should include analysis of the fluid mechanics of the new design, the mechanical properties of the stator and rotor materials, maximum working pressure as well as leakage and backflow of the PCP. Moreover, positive clearance PCP design was explored throughout this work. Interference fit between the rotor and stator should also be investigated, given that elastomers that allow large deformations are usually used in the soft core of commercial stators. Finally, experimental testing of the novel PCP design could also provide valuable insight towards future development and performance evaluation, especially in comparison to commercial PCPs.

\section{References}

1. Lea Jr, J. F., \& Rowlan, L. Gas well deliquification. Gulf Professional Publishing (2019)

2. Nguyen, T., Al-Safran, E., Saasen, A., \& Nes, O. M. (2014). Modeling the design and performance of progressing cavity pump using 3-D vector approach. Journal of Petroleum Science and Engineering, 122, 180-186.

3. Zhou, X. Z., Shi, G. C., Cao, G., Sun, C. L., He, Y., Liu, H., \& Wu, H. A. (2013). Three dimensional dynamics simulation of progressive cavity pump with stator of even thickness. Journal of Petroleum Science and Engineering, 106, 71-76.

4. Chen, J., Liu, H., Wang, F., Shi, G., Cao, G., \& Wu, H. (2013). Numerical prediction on volumetric efficiency of progressive cavity pump with fluidsolid interaction model. Journal of Petroleum Science and Engineering, 109, 12-17.

5. Wu, B., \& Li, X. (2010, January). The special successful PCP applications in heavy oilfield. In SPE progressing cavity pumps conference. Society of Petroleum Engineers.

6. Chen, J., Wang, F., Shi, G., Cao, G., He, Y., Ge, W., ... \& Wu, H. (2015). Finite element analysis for adhesive failure of progressive cavity pump with stator of even thickness. Journal of Petroleum Science and Engineering, 125, 146-153.

7. Delpassand, M. S. (1997, January). Progressing cavity (PC) pump design optimization for abrasive applications. In SPE Production Operations Symposium. Society of Petroleum Engineers.
8. Stewart, M. (2018). Surface Production Operations : Volume IV : Pumps and Compressors. Gulf Professional Publishing.

9. Gravesen, J. (2008). The geometry of the Moineau pump. Computer Aided Geometric Design, 25(9), 792-800.

10. Saveth, K. J., \& Klein, S. T. (1989, January). The progressing cavity pump: principle and capabilities. In SPE Production Operations Symposium. Society of Petroleum Engineers.

11. Noble, E., \& Dunn, L. (2011). Pressure Distribution in Progressing Cavity Pumps: Test Results and Implications for Performance and Run Life.SPE paper 153944. In : (2011) Society of Petroleum Engineers.

12. Paladino, E., Alves de Lima, J., Almeida, R., \& Assmann, B. W. (2008, January). Computational modeling of the three-dimensional flow in a metallic stator progressing cavity pump. In SPE Progressing Cavity Pumps Conference. Society of Petroleum Engineers.

13. Samuel, G. R., \& Saveth, K. (1998, January). Progressing Cavity Pump (PCP): New Performance Equations for Optimal Design. In SPE Permian Basin Oil and Gas Recovery Conference. Society of Petroleum Engineers.

14. Gamboa, J., Aurelio, O., \& Sorelys, E. (2003, January). New approach for modeling progressive cavity pumps performance. In SPE Annual Technical Conference and Exhibition. Society of Petroleum Engineers. 